\title{
COMPROMISO DEPORTIVO, JUICIO DE CONTROL Y BURNOUT EN DOS EQUIPOS DEPORTIVOS FEMENINOS
}

\section{SPORT COMMITMENT, PERCEIVED CONTROL AND BURNOUT IN TWO FEMALE SPORTS TEAMS}

\author{
VerónicaTutte Vallarino \\ Universidad Católica del Uruguay, Uruguay \\ Margarita Suero Girardi \\ Universidad Católica del Uruguay, Uruguay
}

\begin{abstract}
Resumen: El aumento en los casos de bajas deportivas debidas al burnout, así como la disminución del rendimiento, el agotamiento emocional y el abandono en deportistas, han sido algunas de las razones que motivaron nuestro estudio.

Nuestro objetivo es determinar si existen diferencias entre 2 equipos deportivos femeninos a lo largo de una temporada, en función de dos variables (Compromiso Deportivo y Juicio de Control), que podrían predecir la aparición anticipada del burnout. En este sentido, estudiaremos si se producen diferencias entre dichas variables, y si las mismas pueden ser utilizadas como medidas profilácticas para prevenir la posibilidad de desarrollar el síndrome de burnout.

Las muestras estudiadas se componen por 2 equipos de mujeres deportistas de Alto Rendimiento, correspondientes a 10 deportistas de un equipo de básquet profesional de la Liga Femenina de Básquet Española y a 13 deportistas del Seleccionado Nacional de Hockey sobre Césped de Uruguay, ambas entre 18 a 33 años. Todas las participantes fueron evaluadas utilizando el Inventario de Burnout para Deportistas (IBD), las Escalas análogo visuales de Juicio de Control (VAS) y el Cuestionario de Compromiso Deportivo (SCQ).

No se encontraron diferencias significativas en la mayoría de las dimensiones evaluadas entre ambos grupos y en ambas administraciones.
\end{abstract}

Palabras clave: Compromiso Deportivo, Juicio de Control, Burnout, mujeres deportistas.

\begin{abstract}
The increase in dropout cases in sports due to burnout, declining performance, emotional exhaustion and neglect in athletes were some of the reasons for our study.

Our aim is to determine whether differences exist between two female sports teams throughout a season, depending on two variables (Sport Commitment and Perceived Control), which could predict the earlyonset of burnout. In this course of action, we will study if there are differences between these variables, and whether they may be used as prophylactic measures to guard against the possibility of developing burnout syndrome.

The samples are composed by two teams of high performance women athletes, 10 of which integrated a professional basketball team of the Spanish Female Basketball League, and 13 belonged to the Uruguayan National Select Hockey Team, both samples composed by participants between 18 and 33 years of age. All participants were assessed using the Burnout Inventory for Athletes (IBD), the visual analogue scale Perceived Control (VAS) and Sport Commitment Questionnaire (SCQ).

There were no significant differences between the two groups or the two administrations in most dimensions assessed.
\end{abstract}

Keywords: Sport Commitment, Perceived Control, Burnout, sportswomen.

La publicación de este artículo ha sido posible gracias a una parte de la subvención recibida del Fondo Clemente Estable (Proyecto 71/2008- Modalidad III-Joven Investigador) la que sustentó el desarrollo de la metodología de estudio.

\section{INTRODUCCIÓN}

Los estudios sobre el burnout en general comenzaron hace años, pero en la actualidad la frecuencia de este síndrome hace que cada día haya un mayor número de investigaciones al respecto.
El burnout fue definido por distintos autores (Freudenberger, 1974; Maslach y Jackson, 1981; Fender, 1989). Uno de los primeros fue Freudenberger (1974), y se refiere a él como: el sentimiento de fracaso, el agotamiento o la sensación de "volverse exhausto" ante

Correspondencia: Verónica Tutte, Departamento de Ciencias Cognitivas y de la Salud, Facultad de Psicología, Universidad Católica del Uruguay. Correo Electrónico: vtutte@ucu.edu.uy 
excesivas demandas de energía, fuerza espiritual o recursos personales. Maslach y Jackson (1981), lo entienden como un síndrome tridimensional caracterizado por agotamiento emocional, despersonalización y reducida realización personal.

Los deportistas no son una excepción frente a este fenómeno que aparece casi a diario. La intensidad de los entrenamientos y la presión a la que están expuestos los distintos deportistas los convierten en sujetos vulnerables.

Entre los pioneros en la investigación en el deporte está Fender (1989), quien propuso definir el burnout en el deporte como una reacción al estrés de las competencias cuyos síntomas principales son: agotamiento emocional, actitud impersonal hacia los demás y disminución del rendimiento.

Los casos de las bajas deportivas por burnout, la disminución del rendimiento deportivo y el número de abandonos que se producen, son motivo de interés de estudio tanto para el mundo deportivo como para la sociedad en general.

El juicio de control de los distintos deportistas frente a estos eventos estresantes y el compromiso deportivo podrían pronosticar la capacidad de adaptación a la situación o por el contrario de la aparición de diferentes sintomatologías como el burnout, depresión o cualquier otro trastorno.

Otros estudios realizados (Maslach y Jackson, 1986) con deportistas han profundizado en la relación entre el estrés y el burnout, los estados de ánimo negativos y la baja motivación en deportistas que padecen burnout, depresión, inmunidad, entre otros.

Como hemos podido observar hay muchos trabajos relacionados al burnout, pero ninguno de ellos hace referencia al juicio de control y el compromiso deportivo como factores que podrían influir en la posibilidad de desarrollar burnout.

De ahí que consideremos importante continuar y ampliar las investigaciones que ya han sido realizadas, para aportar nuevos conocimientos y herramientas para prevenir la aparición del burnout.

Maslach y Jackson (1986), lograron definir el burnout a partir de los síntomas que lo acompañan, destacando actitudes negativas, insensibilización, culpa, fatiga, insomnio, dolores de cabeza, incremento de la vulnerabilidad a las enfermedades virales, problemas digestivos, abuso de alcohol, drogas, problemas de sociabilidad, falta de deseos de trabajar, autoestima reducida y deterioro de los procesos cognitivos, donde resalta la dificultad para la concentración de la atención.

Siguiendo este planteamiento es que se puede entender las tres dimensiones que caracterizan a este síndrome y es Sarros (1988) quien logra hacer una descripción detallada de las mismas:

- Agotamiento emocional: haría referencia a las sensaciones de sobreesfuerzo físico y hastío emocional que se produce y como consecuencia de las continuas interacciones que los trabajadores deben mantener entre ellos, así como con los clientes.

- Despersonalización: supondría el desarrollo de actitudes y respuestas cínicas hacia las personas a quienes los trabajadores prestan sus servicios.

- Reducida realización personal: conllevaría la pérdida de confianza en la realización personal y la presencia de un autoconcepto negativo como resultado de las situaciones ingratas.

Muchos autores han realizado una búsqueda exhaustiva que logre aclarar las características y síntomas que presenta el burnout, mientras que otros como Garcés de los Fayos y Cantón, (2007) proponen un nuevo modelo para describir el constructo de burnout en deportistas, con la finalidad de proponer estrategias de actuación que incidan de manera adecuada en la potencial solución de este síndrome, tanto en la prevención como en la intervención postsíndrome. Esto es de relevancia puesto que hasta el momento los modelos planteaban las variables que podrían incidir en la aparición del burnout, pero no proponían ni planteaban acciones que podrían resultar efectivas para evitar la aparición de este síndrome.

Por su parte, Nagy y Nagy (1992) y Sandoval (1993) describen las variables predictoras del burnout a través de tres categorías:

- Profesionales u organizacionales: son variables intrínsecas a la organización que pueden por sí mismas generar situaciones aversivas, o bien juegan un papel importante en la interacción continua trabajador - puesto de trabajo.

- Intrapersonales: son variables propias del individuo y hacen referencia a aspectos de su personalidad, sus sentimientos y pensamientos, sus emociones, sus actitudes, etc.

- Interpersonales o ambientales: son variables no relacionadas con el contexto laboral y sí con otros ámbitos que pueden originar fricciones importantes: familia, amistades, estilo de vida, etc. 
Estas variables predictoras del burnout son utilizadas para comprender el desarrollo de este síndrome sin discriminar el entorno donde se enmarque esta situación y aunque puedan ser aplicables al deporte hay otras que son consideradas más específicas para este ámbito.

A continuación les detallaremos algunas de las variables predictoras del Burnout identificadas como más apropiadas y ocurrentes en el contexto deportivo:

- Dificultades que el deportista encuentra con el entrenador

- Altas demandas competitivas

- Excesivas demandas de energía y de tiempo

- Monotonía en el entrenamiento

- Sentimiento de estar apartado

- Carencia de refuerzos positivos

- Aburrimiento

- Falta de habilidades

- Estrategias de afrontamiento poco adaptativas

- Inadaptación entre expectativas originales y logros finales obtenidos

- Falta de apoyo en su grupo de referencia

- Intereses mercenarios de los padres

- Estilo de vida externo

El burnout por tanto, podría aparecer como causa de estas variables. A su vez, dentro de ellas, encontramos algunas como estrategias de afrontamiento poco adaptativas e inadaptación entre las expectativas originales y logros finales obtenidos. Estos conceptos pueden ser desarrollados y comprendidos tanto por el Modelo de Compromiso Deportivo como por la Psicología del Control.

El Compromiso Deportivo es un constructo que pretende identificar los factores que llevan a que alguien persista en una relación interpersonal, organizacional o se mantenga en la actividad deportiva que desarrolla con la finalidad de conocer cuáles son las razones y/o motivos que llevan a un deportista continuar practicando el deporte (Sousa, 2008). Entendemos que el Compromiso Deportivo es un "constructor psicológico que representa el deseo y el propósito de continuar participando en el deporte" (Scanlan y Simons, 1992, pp.6). Así, el abandono podría entenderse como disminución en el Compromiso Deportivo (Schmidt y Stein, 1991).

Para explicar el modelo de Compromiso Deportivo haremos referencia a los trabajos de Scanlan y Simons (1992), ya que creemos que a partir de sus investigaciones podríamos conocer y describir los factores que hacen que una persona se mantenga vinculada a una actividad deportiva.
El modelo que plantean Scanlan, Carpenter, Schmidt, Simons, y Keeler (1993), adaptado de la literatura científica sobre las relaciones amorosas (Rusbult, 1980), propone que el compromiso con la participación deportiva es función del placer individual por el deporte, es decir diversión deportiva, del atractivo de las alternativas fuera del deporte, de la inversión personal en la participación, de las oportunidades de la implicación disponibles por la participación continuada, de las coacciones sociales para continuar participando en el deporte y del apoyo social con el que cuenta el deportista. Este último factor fue posteriormente añadido por los autores al modelo (Scanlan, Russell, Beals, y Scanlan, 2003; Scanlan, Russell, Wilson y Scanlan, 2003).

El Compromiso Deportivo es evaluado en español con el Cuestionario de Compromiso Deportivo (SCQe), adaptación del original Sport Commitment Questionnaire (SCQ) creado por Scanlan et al. (1993), que ha sido adaptado y validado en un estudio previo realizado por Sousa et al. (2007).

Mediante una escala de 32 ítems se pretende evaluar los constructos que antes mencionamos: Compromiso Deportivo (6 ítems), Diversión Deportiva (4 ítems), Alternativas de la Implicación (4ítems), Inversiones Personales (3 ítems), Coacciones Sociales ( 7 ítems) y Oportunidades de la Implicación (4 ítems). Posteriormente, se ha añadido un nuevo constructo, que es el de Apoyo Social (4 ítems), a partir de un estudio cualitativo realizado con un equipo profesional de rugby a través de un sistema de entrevista (Scanlan Collaborative Interview Method, Scanlan, Russell, Wilson, et al., 2003) que ha aportado la introducción de este factor que influye en el compromiso de forma positiva (Sousa, 2008), pero no lo hemos tomado en cuenta en nuestro trabajo.

Consideramos, por tanto, que este instrumento de medida es el más adecuado para nuestro estudio, pues ha sido creado de acuerdo con el modelo de compromiso deportivo, construido por las mismas autoras (Scanlan et al., 1993) y adaptado al castellano presentando un potencial psicométrico adecuado. Así mismo, no encontramos modelos alternativos que permitan entender y evaluar este constructo, por lo que la única opción es la utilización del SCQe.

Por tanto, a través de este modelo pretendemos ahondar y profundizar en los factores que llevan al mantenimiento de la práctica 
deportiva y de esta forma predecir por qué algunos deportistas continúan con la actividad deportiva, mientras que otros abandonan y/o sufren burnout.

Por su parte, la Psicología del Control es entendida como el conjunto de implicaciones derivadas del control conductual, es decir, de la existencia de contingencias entre la conducta del sujeto o de otros individuos y la obtención de determinados resultados (Fernández, Álvarez, Blasco, Doval, Sanz, 1998). El control se refiere a la medida en que un agente provoca, a través de unos medios, de manera intencionada, un resultado deseado y previene o evita resultados no deseados.

Por tanto se podría decir que la sensación de control se asocia a saber lo que se quiere, ser capaz de identificar los modos para conseguirlo, cambiar los objetivos cuando los iniciales no son factibles, ser capaz de reconocer las propias habilidades, saber incrementarlas si son necesarias y decidir cuándo merece la pena ejercer control y cuándo no (Bárez, 2002).

Siguiendo esta línea y trasladándola al contexto deportivo, podríamos decir que un deportista a través del control podría evitar la aparición del burnout y de sus consecuencias frente a una competición o evento que por su importancia genere altos niveles de ansiedad y estrés.

La Figura 1, Esquema de Psicología del Control, (Blasco, 2004) nos presenta el modelo de la Psicología del Control con los factores que lo integran.
El esquema de este modelo puede servirnos para comprender por qué un individuo experimenta una determinada consecuencia (Burnout), y al mismo tiempo evaluar en qué medida el juicio de control puede modular directa o indirectamente el burnout.

El Juicio de Control juega un papel fundamental dentro de este modelo y se refiere a un juicio, opinión o dictamen que el sujeto realiza sobre la contingencia entre sus acciones o las de otros (es decir, entre el agente y los medios) y los resultados.

El Juicio de Control puede ser positivo, es decir se percibe control, con lo que hablaríamos de Control Percibido o Percepción de Control, o puede ser nulo, o lo que es lo mismo: se percibe que no hay control, el individuo cree que un determinado resultado no puede conseguirse porque no existe un agente capaz de desarrollar los medios que dan lugar a dicho resultado (Bárez, Blasco, Fernández - Castro, 2003).

Consideramos importante para clarificar el concepto de Juicio de Control, hacer referencia también a uno de sus componentes: la autoeficacia, que indica que el sujeto valora que el agente (él mismo), es capaz de desarrollar unos medios, que se presupone que permiten conseguir unos determinados resultados deseados por el individuo.

La autoeficacia es una valoración de capacidades de ejecución, es decir una predicción del éxito conductual, mientras que el juicio de control es una valoración de contingencias entre conductas y consecuencias (Bandura, 1982).

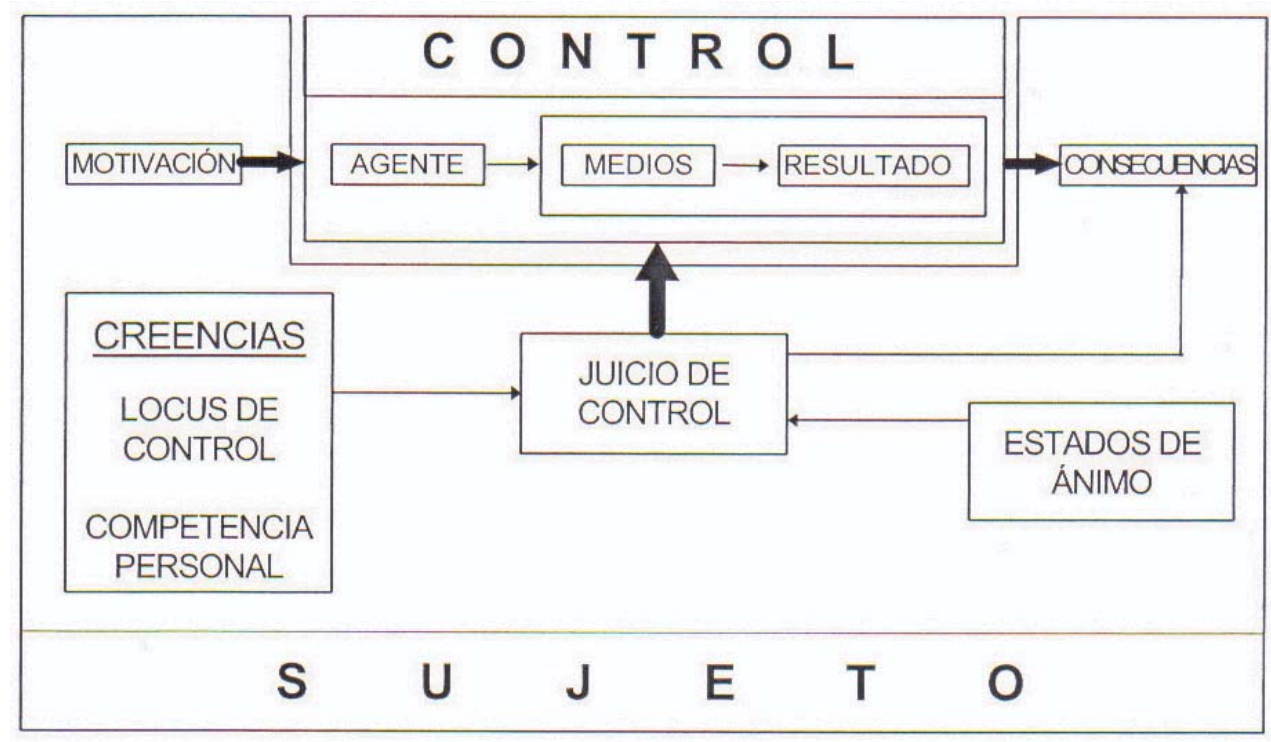

Figura 1: Psicología del Control. 
Por tanto, la autoeficacia lleva implícito el control sobre la ejecución de la conducta, pero no sobre sus consecuencias, mientras que el control percibido o juicio de control incluye tanto el control de estímulos consecuentes como el control que ejerce el individuo sobre la ejecución conductual.

El individuo con Juicio de control valora que el agente (que puede ser él u otra persona), a través de determinados medios, conseguirá determinados resultados. La consecuencia de este razonamiento determinará lo que realmente haga el sujeto ante una situación en concreto y la reacción emocional que expresará. En general se relaciona el Juicio de Control con la manera de afrontar (Carver et al., 2000). De esta manera se mantiene la hipótesis de que en situaciones percibidas como controlables se pondrán en marcha estrategias de afrontamiento activas, que se centrarán en el problema.

Según este esquema el Juicio de Control, en el ámbito del deporte y del burnout jugaría un papel de mediador entre la situación de estrés y el grado de adaptación a la misma y como consecuencia de esto cabría esperar que los individuos con un Juicio de Control alto obtendrían un mayor grado de adaptación frente al evento estresante.

Para que el Juicio de Control pueda ser considerado certero y pueda cumplir esta función de modulador del burnout es necesario que las situaciones que se les presenten a los deportistas sean consideradas motivantes por éstos. Si el acontecimiento al que se enfrenta el atleta no le resulta interesante y estimulante, éste no pondrá en marcha ninguna estrategia ni acción con el fin de ejercer control de la situación.

Por tanto para poder evaluar el Juicio de Control y la relación que éste podría tener con el burnout es que tomaremos en cuenta las variables predictoras del burnout en deportistas (Garcés de los Fayos, 2004), quitando alguna de ellas por considerar que eran reiterativas, resultando de tal modificación 10 variables (de 12 iniciales) consideradas predictoras. Estas variables cumplirían la condición -antes señalada- de implicar motivacionalmente a los individuos y son las siguientes:

- Dificultades que el deportista encuentra con el entrenador

- Demandas competitivas y de tiempo

- Sentimiento de estar apartado

- Carencia de refuerzos positivos

- Aburrimiento

- Falta de habilidades
- Estrategias de afrontamiento negativas

- Falta de apoyo

- Intereses mercenarios de los padres

- Estilo de vida externo

Para la evaluación del burnout, la mayoría de los autores utilizan el Maslach Burnout Inventory: MBI (Maslach y Jackson, 1981). Son numerosas las investigaciones que apoyan la validez, fiabilidad y estructura factorial del MBI, consolidándose como el instrumento de elección para las distintas investigaciones que procuran profundizar en este síndrome. Autores como Cherniss (1992), Leiter (1991), Golembiewski (1993) y otros no sólo aceptan la fiabilidad y validez del $\mathrm{MBI}$, sino que también concluyen que la estructura factorial queda confirmada.

El MBI es un inventario de 22 ítems que se divide en tres subescalas, cada una de las cuales evalúa las tres dimensiones que supuestamente configuran el constructo burnout: Agotamiento Emocional (9 ítems), Despersonalización (5 ítems) y Reducida Realización Personal (8 ítems). De cada una de estas escalas se obtiene una puntuación baja, media o alta (que coincide con los tres tercios en los que se distribuye la población normativa) que permite caracterizar la mayor o menor frecuencia del burnout.

Para nuestro estudio concretamente, nos vamos a referir al Inventario de Burnout en Deportistas (IBD) que Garcés de los Fayos (1999) adapta a partir de una modificación de la estructura teórica del Maslach Burnout Inventory (Maslach y Jackson, 1981), partiendo de un inventario con 22 ítems a los que la persona debe responder mediante una puntuación de frecuencia según una escala Likert de cinco opciones.

Garcés de los Fayos (2004) comprobó que, debido a que la forma de agruparse de los ítems, se confirma el mantenimiento de los principios teóricos y empíricos de Maslach y Jackson (1981) para la creación y desarrollo del Inventario de Burnout en Deportistas, como un instrumento adecuado para medir burnout en esta población.

La fiabilidad del IBD (Garcés de los Fayos, 1999) medida mediante el coeficiente alfa es de 0.65 . En cuanto a su validez factorial y consistencia interna, queda demostrada en Garcés de los Fayos, García y Martínez (1998).

García Ucha (2002), utilizó el IBD, con deportistas cubanos, confirmando la fiabilidad y validez de esta herramienta. 
Un deportista padece burnout cuando sus puntuaciones se sitúan en Agotamiento Emocional y Despersonalización a partir del percentil 66 y por debajo del percentil 33 en Reducida Realización Personal, que se corresponden con las calificaciones de burnout severo de Maslach y Jackson (1981). Puntuaciones en Agotamiento Emocional y Despersonalización por debajo del percentil 33, y por encima del percentil 66 en Reducida Realización Personal fueron consideradas como representativas de deportistas sin burnout (Garcés de los Fayos, 2004), dejando el resto de las puntuaciones en una zona ambigua que no permite hacer predicciones claras sobre cómo evolucionarán los atletas.

Creemos entonces que tomando en cuenta las variables predictoras, que son elementos clave para un deportista de elite y por tanto consideradas importantes para él, podríamos llegar a estimar una posible relación entre el Juicio de Control, el Compromiso Deportivo y el Burnout evaluado a partir del IBD, en la cual dependiendo del control que una persona crea tener frente a situaciones ambiguas, difíciles o novedosas tendrá así, mayor o menor posibilidad de que aparezca el síndrome (García Ucha, 2002).

\section{MÉTODO}

\section{PARTICIPANTES}

Este estudio cuenta con dos muestras distintas de mujeres deportistas de Alto Rendimiento. La muestra 1 corresponde a diez deportistas que integran un equipo de básket profesional de la Liga Femenina de Básquet Española, de edades comprendidas entre los 18 y 33 años. La muestra 2 abarca trece deportistas que integran el Seleccionado Nacional de Hockey sobre Césped de Uruguay. Las edades de estas participantes comprenden entre los 18 y 32 años.

La actividad semanal promedio para ambos grupos es de 20 horas de entrenamiento y dos horas de partido.

\section{MATERIALES}

Se utilizaron tres escalas para obtener la información requerida acorde con los objetivos propuestos.

1-Inventario de Burnout para Deportistas (IBD): Adaptación realizada por Garcés de los Fayos (1999) del Maslach Burnout Inventory (Maslach y Jackson, 1981).
Dicho cuestionario consta de 30 ítems, que se subdividen en tres dimensiones: Agotamiento Emocional, Despersonalización y Reducida Realización Personal. Se utiliza una escala del tipo Likert que va del 1 "nunca lo he pensado o sentido" a 5 "lo pienso o siento todos los días". La interpretación de las puntuaciones de la escala se realiza a partir de los percentiles establecidos: un deportista padece burnout cuando sus puntuaciones se sitúan en Agotamiento Emocional y Despersonalización a partir del percentil 66 y por debajo del percentil 33 en Reducida Realización Personal, que se corresponden con las calificaciones de burnout severo de Maslach y Jackson (1981). Puntuaciones en Agotamiento Emocional y Despersonalización por debajo del percentil 33, y por encima del percentil 66 en Reducida Realización Personal fueron consideradas como representativas de deportistas sin burnout (Garcés de los Fayos, 2004), dejando el resto de las puntuaciones en una zona ambigua que no permite hacer predicciones claras sobre cómo evolucionarán los atletas.

2-Escalas análogo visuales de Juicio de Control: Diez ítems que evalúan la percepción de control que el sujeto tiene frente a distintas situaciones a las que se tiene que enfrentar en el contexto deportivo. Se contesta a través de escalas que van del 0 "poco capaz" al 100 "muy capaz". Estos ítems se han elaborado para esta investigación a partir de la experiencia obtenida en un trabajo anterior (Tutte, 2005). No existe baremación para los juicios y por tanto hemos creado tres niveles para comprender si existe un adecuado, bajo o alto nivel de control. Para ellos tomamos como referente los estudios realizados por el GIES (Grupo de Investigación en Estrés y Salud) y que hemos aplicado también en un trabajo anterior (Tutte, 2005). Estos criterios que proponían un valor entre 60 y 70 como adecuado, >70 como un valor alto de control y $<$ de 60 como un valor bajo de control.

3-Cuestionario de Compromiso Deportivo (SCQ): Utilizaremos la versión traducida al castellano (Sousa, Torregrosa, Viladrich, Villamarín, Cruz, 2007) del Sport Commitment Questionnaire (Scanlan, Carpenter, Schmidt, Simons, Keeler, 1993). Dicho cuestionario consta de 32 ítems, que se subdividen en 7 dimensiones distintas (Scanlan et al.,1993). Cada ítem se contesta en una escala del tipo Likert del 1 al 5, que fluctúa desde la valoración "muy en desacuerdo" hasta "muy de acuerdo". 
La fiabilidad de cada una de estas dimensiones es de alfa $=.76$ para compromiso deportivo, alfa $=.88$ en diversión deportiva. Alfa $=.66$ en alternativas a la implicación, alfa $=.41$ en inversiones personales, alfa $=.80$ en coacciones sociales y alfa $=.52$ en oportunidades a la implicación, aludiendo a una adecuada consistencia interna en tres de las seis dimensiones: compromiso, diversión, coacciones sociales y alternativas a la implicación que fue considerada aceptable aunque marginal (Sousa, Torregrosa, Viladrich, Villamarín y Cruz, 2007).

\section{PROCEDIMIENTO}

Esta investigación se desarrolló para cada uno de los equipos en períodos diferentes, tomando en cuenta el comienzo de la temporada deportiva.

En Octubre del 2004 nos pusimos en contacto con un club profesional de básket femenino de la Liga Española de Básket, a quien le presentamos nuestro trabajo.

Las jugadoras decidieron colaborar firmando un consentimiento en donde aceptaban participar de esta investigación. Al tratarse de un estudio longitudinal se pautó con las deportistas dos instancias distintas para poder administrar las técnicas, un período inicial a comienzo de temporada (noviembre y diciembre de 2004) y un segundo período al cabo de tres meses (febrero y marzo de 2005).

Mientras que con nuestra segunda muestra nos contactamos en diciembre de 2006 y luego de informar las características del trabajo al cuerpo técnico y las jugadoras, integrantes de un seleccionado nacional de Hockey sobre Césped, ellas también aceptaron ser parte de este trabajo.

En este sentido, se elaboró un calendario respetando las características del procedimiento que antes habíamos realizado con el equipo español. Se llevó a cabo una primera administración a comienzos de la temporada (febrero de 2007) y una segunda instancia tres meses más tarde, a finales de temporada (mayo de 2007).

Una vez obtenida la información a partir de las técnicas empleadas es que procedimos a la corrección y al análisis de dichos datos.

\section{DISEÑO}

Este estudio responde a un diseño no experimental, descriptivo, longitudinal, de grupos comparativos.

\section{ANÁLISIS DE RESULTADOS}

Para poder analizar sí existen diferencias a lo largo del tiempo dentro de cada deporte y entre los mismos, es que procedimos a realizar un análisis de la varianza de medidas repetidas (MANOVA), (Pardo, Ruiz-Díaz, 2002), 2inter (deportes: Básket y Hockey) x 2 intra (tiempo: inicio-final). En el caso de la variable dependiente Burnout, se utilizó tablas de contingencia y Chi cuadrado para poder analizar si existían o no diferencias significativas entre el inicio y el final de la temporada.

\section{RESULTADOS}

Los resultados indican que en el inicio de la temporada deportiva, la gran mayoría de los JC han obtenido puntuaciones adecuadas ( $>$ de 70 puntos, y $<40$ para el JC1) en ambos deportes a excepción de JC1 "relación con el entrenador" para las balocentistas que presentan puntuaciones ligeramente más elevadas a lo esperado, indicando una menor capacidad para manejar la influencia negativa que el entrenador puede ejercer sobre su rendimiento deportivo, mientras que no sucede lo mismo con las jugadoras de Hockey, pues se encuentran dentro del límite adecuado. Lo mismo ocurre con el JC4 "Aislamiento" y en el JC5 "Carencia de reforzamientos" que presenta descendidas puntuaciones $(<60)$ en ambos deportes.

EI JC10 "Estilo de vida externo" presenta elevadas puntuaciones en ambos deportes, sin embargo son las jugadores de básket $(M=93,62 ; D S=5,25)$ las que presentan una puntuación más elevada y significativa.

El análisis de la varianza de medidas repetidas (MANOVA), fue significativo para el JC3 "Aburrimiento" que presenta diferencias en los resultados a partir de la interacción del tiempo y el deporte.

El valor de Lambda de Wilks $=.816 \mathrm{~F}$ $(\mathrm{Sig}=.036)$ revela que el efecto del tiempo y el deporte obtienen niveles críticos por debajo de 0.05 por lo que puede concluirse que existe un efecto significativo de la interacción, siendo que en el inicio (pre) de la temporada no existían diferencias estadísticamente significativas entre el básket y el hockey, mientras que sí se presentan diferencias en el final (post) de la misma, obteniendo las balocentistas puntuaciones menores en relación a las jugadoras de hockey. 
En el JC10 "Estilo de vida externo" también aparecen diferencias estadísticamente significativas que revelan los efectos de la interacción del tiempo y el deporte, a partir del valor Lambda de Wilks=.824 F (Sig=.041; $p<.05)$. Al inicio encontramos diferencias entre los deportes: básket y hockey, mientras que esas diferencias desaparecen en el final de la temporada deportiva.

Les presentamos la Tabla 1: que presenta la media obtenida en los 10 Juicios de Control en el inicio y el final de la temporada deportiva. puntuaciones (>3.5) en la subescala de Compromiso Deportivo, Alternativas a la Implicación, Inversiones Personales y en Oportunidades a la Implicación. Así mismo, tanto las balocentistas como las jugadoras de hockey presentan puntuaciones adecuadas (entre 2.5 y 3.5 ) en Diversión Deportiva y bajas puntuaciones en Coacciones Sociales (<2.5), siendo los resultados similares para los dos equipo deportivos.

Sin embargo el paso del tiempo produce modificaciones dentro de cada deporte y entre ellos.

\begin{tabular}{|c|c|c|c|c|c|}
\hline & \multicolumn{2}{|c|}{ Básket } & \multicolumn{2}{|c|}{ Hockey } \\
\hline & & $\mathbf{M}$ & DT & $\mathbf{M}$ & DT \\
\hline \multirow{2}{*}{ JC1: Relación con el entrenador } & 1 & 47,51 & 33,52 & 40,55 & 25,45 \\
\hline & 2 & 55,40 & 30,67 & 54,79 & 18,64 \\
\hline \multirow{2}{*}{ JC2: Exigencia de la competición } & 1 & 84,00 & 19,30 & 75,23 & 14,10 \\
\hline & 2 & 77,80 & 27,12 & 79,71 & 14,61 \\
\hline \multirow{2}{*}{ JC3: Aburrimiento } & 1 & 69,11 & 18,10 & 74,64 & 14,42 \\
\hline & 2 & 53,80 & 28,24 & 80,14 & 13,68 \\
\hline \multirow{2}{*}{ JC4: Sentirse aislado } & 1 & 38,95 & 33,30 & 38,23 & 27,77 \\
\hline & 2 & 21,80 & 14,08 & 37,71 & 24,60 \\
\hline \multirow{2}{*}{ JC5: Carencia de reforzamientos } & 1 & 39,32 & 27,25 & 55,36 & 20,85 \\
\hline & 2 & 42,00 & 24,53 & 47,86 & 21,11 \\
\hline \multirow{2}{*}{ JC6: Autoeficacia en el juego } & 1 & 75,78 & 15,10 & 74,09 & 16,44 \\
\hline & 2 & 76,20 & 16,19 & 81,57 & 15,67 \\
\hline \multirow{2}{*}{ JC7: Superación de dificultades } & 1 & 74,56 & 14,10 & 79,09 & 15,61 \\
\hline & 2 & 74,50 & 14,66 & 79,71 & 14,90 \\
\hline \multirow{2}{*}{ JC8: Apoyo de los padres } & 1 & 96,33 & 4,31 & 90,59 & 19,28 \\
\hline & 2 & 97,10 & 3,78 & 92,79 & 8,91 \\
\hline \multirow{2}{*}{ JC9: Expectativas de los padres } & 1 & 14,61 & 30,67 & 16,55 & 12,66 \\
\hline & 2 & 13,40 & 30,53 & 17,29 & 12,21 \\
\hline \multirow{2}{*}{ JC10: Estilo de vida externo } & 1 & 93,62 & 5,25 & 81,18 & 20,57 \\
\hline & 2 & 77,80 & 25,44 & 86,29 & 13,00 \\
\hline
\end{tabular}

Tabla 1: Medias y desvíos de las subescalas de los Juicios de Control, en la primera y segunda administración

La siguiente Tabla 2, presenta los valores medios obtenidos a partir del Cuestionario de Compromiso Deportivo (SCQe) tanto en el inicio como en el final de la temporada.

Ambos equipos deportivos presentan en el inicio de la temporada deportiva elevadas
Así lo indica el análisis de la varianza de medidas repetidas (MANOVA).

Dentro del Modelo de Compromiso Deportivo, las subescalas Compromiso Deportivo y Diversión Deportiva presentan diferencias significativas en la interacción tiempo y deporte. 
En el compromiso deportivo el valor Lambda de Wilks=.799 F (Sig=.032; $p<.05)$ indica que el efecto de la interacción tiempo y deporte es significativa.

\begin{tabular}{|lc|c|c|c|c|}
\hline \multirow{2}{*}{ Compromiso Deportivo } & & \multicolumn{2}{|c|}{ Básket } & \multicolumn{2}{c|}{ Hockey } \\
\cline { 3 - 6 } & 2 & 3,48 & 0,41 & 3,46 & 0,41 \\
\hline \multirow{2}{*}{ Diversión Deportiva } & 1 & 3,06 & 0,40 & 2,85 & 0,38 \\
& 2 & 2,65 & 0,36 & 2,87 & 0,30 \\
\hline Alternativas a la & 1 & 3,66 & 0,46 & 3,69 & 0,34 \\
Implicación & 2 & 3,23 & 0,51 & 3,44 & 0,59 \\
\hline \multirow{2}{*}{ Inversiones Personales } & 1 & 4,12 & 0,71 & 4,22 & 0,46 \\
& 2 & 4,04 & 0,59 & 4,08 & 0,75 \\
\hline Coacciones Sociales & 1 & 2,48 & 0,58 & 2,56 & 0,33 \\
& 2 & 2,48 & 0,41 & 2,52 & 0,33 \\
\hline Oportunidades a la & 1 & 3,48 & 0,32 & 3,38 & 0,65 \\
Implicación & 2 & 3,40 & 0,43 & 3,33 & 0,54 \\
\hline
\end{tabular}

Tabla 2: Medias y desvíos de las subescalas del Compromiso Deportivo, en la primera y segunda administración

En el inicio de la temporada no existían diferencias entre los valores obtenidos por las jugadoras de básket y por las jugadoras de hockey.

Sin embargo sí se presentan diferencias entre las mismas al final de la temporada, siendo las jugadoras de hockey las que presentan puntuaciones más elevadas en relación al compromiso.

Por su parte, la diversión deportiva presenta también diferencias significativas a partir del valor de Lambda de Wilks=.840 F (Sig=.059) y un nivel crítico asociado próximo a la significación $\mathrm{p}=.05$, indicando que el efecto de la interacción tiempo y deporte es significativo.
En el inicio no existían diferencias entre el básket y el hockey, pero sí existen en el final, donde las jugadoras de hockey obtienen puntuaciones más elevadas en relación al inicio de la temporada y en relación a las balocentistas.

Por último, la subescala Alternativas a la Implicación presenta diferencias estadísticamente significativas en el tiempo, en el inicio (pre) y en el final (post) de la temporada, dentro de cada deporte. El valor Lambda de Wilks $=.782 \mathrm{~F}$ $(\mathrm{Sig}=.025 ; p<.05)$ indica que el efecto del tiempo es significativo, logrando puntuaciones más bajas al final de la temporada en ambos equipos.

La Tabla 3, presenta la distribución de la frecuencia de casos en los tres niveles de Burnout para cada deporte y en las dos administraciones (inicio y final de la temporada deportiva).

En el inicio de la temporada deportiva el 70 $\%$ de las balocentistas y el $85 \%$ de las jugadoras de hockey presentaban algún síntoma de burnout, indicando que en ambos deportes solo un porcentaje menor se encontraba sin síntomas de burnout. No encontramos diferencias estadísticamente significativas entre los deportes y los niveles de burnout (sin síntomas y con síntomas de burnout).

Sin embargo, en el final de la temporada se observa una evolución distinta para cada uno de los deportes. El básket presenta un aumento en el porcentaje de casos con síntomas de burnout (aumenta un 10\%), mientras que el hockey presenta una mejora hacia el final de la temporada deportiva, a partir de un menor número de casos con síntomas de burnout (un $15 \%$ menos).

A pesar de la distinta evolución que presenta los dos equipos no encontramos diferencias estadísticamente significativas entre los resultados obtenidos en ambos deportes y entre el inicio y el final de la temporada.

\begin{tabular}{|lc|c|c|}
\hline $\begin{array}{c}\text { Agotamiento Emocional } \\
\text { Tiempo }\end{array}$ & Básket & Hockey \\
\hline Sin Burnout & 1 & $3(30 \%)$ & $2(15.4)$ \\
Algún Síntoma & 2 & $2(20.0 \%)$ & $4(30.8 \%)$ \\
& 1 & $7(70 \%)$ & $11(84.6 \%)$ \\
& 2 & $8(80.0 \%)$ & $9(69.2 \%)$ \\
\hline
\end{tabular}

Tabla 3: Frecuencia de deportistas en la dimensión Agotamiento Emocional en el inicio y en el final de la temporada. 
En la siguiente Tabla 4: presentaremos la frecuencia de casos que se encuentra en la dimensión Despersonalización para ambos deportes y en las dos instancias en que fueron administrados los cuestionarios.

\begin{tabular}{|lr|l|l|}
\hline $\begin{array}{c}\text { Despersonalización } \\
\text { Tiempo }\end{array}$ & Básket & \multicolumn{1}{|c|}{ Hockey } \\
\hline Sin Burnout & 1 & $8(80 \%)$ & $12(92.3 \%)$ \\
& 2 & $8(80.0 \%)$ & $13(100.0 \%)$ \\
Algún Síntoma & 1 & $2(20 \%)$ & $1(7.7 \%)$ \\
& 2 & $2(20.0 \%)$ & 0 \\
\hline
\end{tabular}

Tabla 4: Frecuencias de Burnout en la dimensión Despersonalización en el inicio y en el final de la temporada deportiva.

A diferencia de lo ocurrido con la dimensión Agotamiento Emocional, aquí encontramos que la mayoría de las deportistas de ambos equipos no presentan síntomas de burnout para la dimensión Despersonalización. Los casos que puntúan algún síntoma se encuentran entre un 8\% para el hockey y un 20\% para el básket.

Hacia el final de la temporada deportiva, observamos que el porcentaje de casos con síntomas de burnout para la dimensión despersonalización se mantiene (20\%), mientras que no existen casos de burnout en esta dimensión para el hockey, indicando una mejora que las coloca en una situación sin riesgo de burnout. No existen diferencias significativas entre los deportes, ni entre el inicio y el final de la temporada deportiva.

Por último, presentamos la Tabla 5: que presenta la frecuencia de casos con y sin síntomas de burnout para la dimensión Reducida Realización Personal, en ambos deportes y en el inicio y final de la temporada deportiva.

En ambas administraciones, la mayoría de las deportistas no presentan síntomas de burnout en esta dimensión.

En el caso de las balocentistas un 90\% no tiene síntomas de burnout, mientras que un $77 \%$ de las jugadoras de hockey se encuentran libres de la presencia de síntomas.

En la segunda administración aumenta el número de casos con síntomas de burnout para la dimensión Reducida Realización Personal, en ambos equipos, pues observamos que al final de la temporada un $20 \%$ de jugadoras de básket desarrolla algún síntoma, al igual que un $38.5 \%$ de las jugadoras de hockey.

\begin{tabular}{|lr|l|l|}
\hline $\begin{array}{c}\text { Reducida Realización } \\
\text { Personal } \\
\text { Tiempo }\end{array}$ & Básket & Hockey \\
\hline Sin Burnout & 1 & $9(90 \%)$ & $10(76.9 \%)$ \\
& 2 & $8(80.0 \%)$ & $8(61.5 \%)$ \\
Algún Síntoma & 1 & $1(10 \%)$ & $3(23.1 \%)$ \\
& 2 & $2(20.0 \%)$ & $5(38.5 \%)$ \\
\hline
\end{tabular}

Tabla 5: Frecuencias de Burnout en la dimensión Reducida Realización Personal al inicio y al final de la temporada.

Sí bien hay un aumento en el porcentaje de casos con síntomas de burnout hacia el final de la temporada, esas diferencias no son significativas ni entre el inicio y el final, ni tampoco entre ambos deportes.

\section{DISCUSIÓN Y CONCLUSIONES}

Comenzando por el burnout, podemos observar que al inicio de la temporada no hay ningún caso que cumpla los criterios de burnout, aún cuando una gran mayoría de casos se encuentran dentro de la zona ambigua, principalmente en la dimensión de Agotamiento Emocional. Esto está de acuerdo, en líneas generales, con los resultados que muestra la literatura. No obstante, lo que sí es interesante es la posibilidad de constatar los cambios a lo largo de la temporada, ya que al final de la misma aparece una leve mejora en la dimensión Agotamiento Emocional en el equipo de hockey no así en las balocentistas, pues aumenta el número de casos con burnout. Si bien podemos observar una evolución distinta en los dos equipos a lo largo de la temporada, la diferencia encontrada no llega a ser significativa. Lo mismo ocurre con la dimensión Despersonalización, donde nuevamente encontramos una mejora en las jugadoras de hockey hacia el final de la temporada mientras que las jugadoras de basket mantienen el mismo porcentaje que en el inicio. Sin embargo en el final de la temporada deportiva ambos equipos presentan un mayor número de casos que puntúan burnout para la dimensión Reducida Realización Personal. Esto podría indicar que la aparición de síntomas de burnout no necesariamente conduce a una situación crónica o a un empeoramiento, sino que puede darse un retorno a niveles normales. 
A través del análisis de la varianza de medidas repetidas (MANOVA) observamos que se encuentran algunas diferencias estadísticamente significativas a lo largo de la temporada deportiva, dentro de cada categoría o entre las mismas, ya sea por el efecto del tiempo, es decir en el inicio de la temporada o al final, y/o por el efecto de la interacción tiempo-deporte.

El JC3 "Aburrimiento" presenta diferencias en la interacción tiempo y deporte, pues al inicio de la temporada no se encontraban diferencias significativas y sí aparecen en el final de la misma, resultando ser las mujeres balocentistas las que obtienen una puntuación mayor, indicando así que a medida que trascurre la temporada las jugadoras de básket se sienten menos capaces de sobrellevar el aburrimiento de las prácticas y partidos que antes, mientras que sucede lo contrario con las jugadoras de hockey, que sienten más capaces hacia el final de la temporada.

Por su parte, también encontramos diferencias en el JC10 "Estilo de Vida Externo". Si bien en el inicio de la temporada ambos equipos presentaban elevadas puntuaciones, son las balocentistas la que obtienen una mayor puntuación generando así diferencias significativas con las jugadoras de hockey. Estas diferencias dejan de existir hacia el final de la temporada, pues las jugadoras de básket descienden sus puntuaciones mientras que las jugadoras de hockey aumentan las mismas. Una alta puntuación en este juicio indicaría que los deportistas de alto rendimiento, independientemente del nivel y experiencia que tengan, saben que la profesionalización obliga a restringir la vida social en todo aquello que pueda afectar negativamente al rendimiento. Sin embargo, observamos que la percepción de control de este juicio en ambos deportes es distinta a lo largo de la temporada, pues las balocentistas sienten una menor capacidad para limitar su vida social en el final de la temporada, mientras que las jugadoras de hockey mejoran, presentando una mayor capacidad hacia el final.

Respecto a los aspectos valorados dentro de la escala de Compromiso Deportivo, debe destacarse que hay pequeñas diferencias entre la muestra de hockey uruguaya y la muestra catalana de baloncesto en el sentido de que el compromiso aumenta a lo largo de la temporada en las jugadoras de hockey pero no sucede lo mismo con la balocentistas. A su vez la diversión deportiva no disminuye, o lo hace muy ligeramente, en el caso de las jugadoras de basket. Esto podría estar evidenciando la existencia de diferencias culturales o bien una situación particular debida a las características de la dinámica de una institución deportiva concreta (protocolo de actividades a realizar, posibilidades de promoción, características del entrenador). Serían necesarios más trabajos que analizasen las posibles causas de estas diferencias. No obstante, parece importante considerar que compromiso y diversión son dos facetas complementarias en la prevención del síndrome de burnout.

En cuanto a las Alternativas para estar implicado con la actividad deportiva, encontramos que disminuyen en ambos grupos hacia el final de la temporada deportiva, lo que podría estar evidenciando cierto cansancio y agotamiento que no permite valorar las opciones no deportivas de igual forma que en el inicio de la temporada.

Finalmente consideramos importante señalar dentro de las limitaciones de nuestro estudio algunas de las desigualdades encontradas, a varios niveles, que podrían incidir en las inferencias realizadas a partir de los resultados. En principio, las desigualdades propias de las muestras comparadas, debido a que si bien ambas eran muestras de mujeres deportistas de alto rendimiento, los deportes practicados eran distintos. Segundo, los diferentes períodos de tiempo en los cuales fueron realizadas las administraciones también pudieron haber afectado los resultados. Tercero, la ubicación geográfica distinta, tratándose de países completamente diferentes.

\section{REFERENCIAS}

Bandura, A. (1982) Self - efficacy mechanism in human agency. American Psychologist, 37 (2), 122-147.

Bárez, M. (2002) Relación entre la percepción de control y adaptación a la enfermedad en pacientes con cáncer de mama. Tesis Doctoral no publicada. Universitat Autónoma de Barcelona. Bellaterra: Barcelona.

Bárez, M., Blasco, T., Fernández - Castro, J. (2003) La inducción de sensación de control como elemento fundamental de la eficacia de las terapias psicológicas en pacientes de cáncer. Anales de Psicología, 19 (2), 235246.

Blasco, T. (2004) Asignatura: Avances en Psicología de la Salud. Apuntes de clase. Programa de Doctorado Psicología del Aprendizaje Humano. Universitat Autónoma de Barcelona, Barcelona.

Carver, C., Harris, S., Lehman, J. M., Durel, L. A., Antoni, M. N., Spencer, S. M. y Pozo, C. (2000) How important is the perception of personal control? Studies of early stage breast cancer patient. Personality and Social Psychology Bulletin, 26, 139-149.

Cherniss, C. (1980) Professional burnout in human service organizations. Nueva York: Praeger.

Fender, L. K. (1989)Athlete burnout: Potential for research and intervention strategies. Sport Psychologist, 3 (1), 63-71. 
Fernández - Castro, J., Álvarez, M., Blasco, T., Doval, E y Sanz, A. (1998) Validación de la Escala de Competencia Personal de Wallston: Implicaciones en el estudio del estrés. Ansiedad y estrés, 4, 31-41.

Fernández - Castro, J., Doval, E., Edo, S. y Santiago, M. (1992) L 'estrés docent del mestres de Catalunya. Barcelona. Informe del Departament d'Ensenyament.

Freudenberger, H. J. (1974). Staff burnout. The Journal of Social Issues, 30 (1), $159-166$.

Garcés de los Fayos, E. J. (1999) Burnout en deportistas: un estudio de la influencia de variables de personalidad, sociodemográficas y deportivas en el síndrome. Tesis Doctoral no publicada. Murcia: Universidad de Murcia.

Garcés de los Fayos, E. J. (2004) Burnouten deportistas. Propuestadeunsistemadeevaluaciónintegral. Madrid:EOS.

Garcés de los Fayos, E. J. y Cantón, E. (2007) Burnout en deportistas. Un modelo teórico-descriptivo del burnout en deportistas: Una propuesta tentativa. Revista Psicológica, 91, pp. 12-22.

Garcés de los Fayos, E. J. y Vives Benedicto, L. (2003) Hacia un modelo teórico explicativo del burnout en deportistas: Una propuesta integradora. Edupsykhé, 2, 221-242.

Garcés de los Fayos, E. J. y Vives Benedicto, L. (2004) Incidencia del síndrome de burnout en el perfil cognitivo en jóvenes deportistas de alto rendimiento. Cuadernos de Psicología del Deporte, 4 (1-2), 30-43.

Golembiewski, R. T et al (1993) Transnacional perspectivas on job burnout: Replication of phase model results among Japanese respondents. The International Journal of Organizational Analysis, 1 (1), 7-27.

Leiter, M. P. (1991) The dream denied: Professional burnout and the contraints of human services organizations. Canadian Psychology, 32 (4), 547-558.

Maslach, C. y Jackson, S. E. (1981) MBI: Maslach Burnout Inventory. Manual. Palo Alto: University of California. Consulting Psychologists Press.

Maslach, C. y Jackson, S. E. (1986) MBI: Maslach Burnout Inventory. Manual Research Edition. Palo Alto:University of California, Consulting Psychologists Press.

Nagy, S. y Nagy, M.C. (1992) Longitudinal examination of teachers burnout in a school district. Psychological Reports, 7 (2), 523-531.

Pardo, A. (2008). Modelos Lineales: Guía docente. Manuscrito no publicado, Universidad Autónoma de Madrid, Facultad de Psicología, Madrid: España.

Rusbult, C. E. (1980) Commitment and satisfaction in romantic associations: A test of the investment model.Journal of Experimental Social Psychology, 16, 172-186. Sandoval, J. (1993) Personality and burnout school psychologists. Psychology in the schools, 30 (4), 321-326.
Sarros, J. C. (1988) Administrator burnout: Finding and future directions. The Journal of Educational Administration, 26 (2), 184-196.

Scanlan, T. K, Russell, D. G., Beals, K. P. y Scanlan, L. A. (2003) Project on elite athlete commitment (PEAK) : II. A direct test and expansion of the Sport Commitment model with elite amateur sportsmen. Journal of Sport and Exercise Psychology, 25, 377-401.

Scanlan, T. K, Russell, D. G., Wilson, N. C. y Scanlan, L. A. (2003) Project on elite athlete commitment (PEAK) I. Introduction and Methodology. Journal of Sport and Exercise Psychology, 25, 360-376. Smith, R. E. y Smoll, F. L. (1997ª). Coaching the coaches: Youth Sports as a scientific and applied behavioural setting. Current Directions in Psychological Science, 6, 16-21.

Scanlan, T. K. y Simons, J. P. (1992) The construct of sport enjoyment. In G.C. Roberts (Ed). Motivation in Sport and Exercise (pp. 199-215). Champaign, IL: Human Kinetics.

Scanlan, T. K., Carpenter, P. S., Schmidt, G. W., Simons, J. P. y Keeler, B. (1993) An introduction to the Sport Commitment Model. Journal of Sport and Exercise, 15, 1-15.

Scanlan, T. K., Simons, J. P., Carpenter, P. S., Schmidt, G. W., y Keeler, B. (1993) The sport commitment model: Measurement development for the youth-sport domain. Journal of Sport and Exercise Psychology, 15, 16-38.

Scanlan, T. K., Simons, J. P., Carpenter, P. S., y Schmidt, G. W. (1993) The Sport Commitment Model: Measurement development for the young - sport domain. Journal of Sport and Exercise Psychology, 15, 16.38.

Scanlan, T.K., (1986) Competitive stress in children. In M.R. Weiss, \& D. Gould (Eds.), Sport for children and youth (pp. 113-118). Champaign, IL: Human Kinetics.

Schmidt, G. W. y Stein, G. L. (1991) Sport Commitment: A model integrating enjoyment, dropout, and burnout. Journal of Sport and Exercise Psychology, 13 (3), 254265.

Sousa, C., Smith, R. y Cruz, J. (2008) An individualized behavioral goal-setting program for coaches. Journal of Clinical Sport Psychology, 2, 258-277.

Sousa, C., Torregrosa, M.., Viladrch, C., Villamarín, F. y Cruz, J. (2007) The commitment of young soccer players. Psicothema, 19 (2), 256-262.

Tutte, V. (2005) Factores que inciden en la aparición del burnout en un equipo femenino de baloncesto. Trabajo de investigación no publicado. Universitat Autónoma de Barcelona. Bellaterra, Barcelona. 